\title{
Optimal Portfolios with Haezendonck Risk Measures
}

\author{
Fabio Bellini, Emanuela Rosazza Gianin
}

December 20, 2007

\begin{abstract}
We deal with the problem of the practical use of Haezendonck risk measures (see Haezendonck and Goovaerts [8], Goovaerts et al. [7], Bellini and Rosazza Gianin [4]) in portfolio optimization. We first analyze the properties of the natural estimators of Haezendonck risk measures by means of numerical simulations and point out a connection with the theory of $M$-functionals (see Hampel [9], Huber [11], Serfling [18]) that enables us to derive analytic results on the asymptotic distribution of Orlicz premia and on their influence functions. We then prove that as in the CVaR case (see Rockafelar and Uryasev [16], [17], Bertsimas et al. [6]) the mean/Haezendonck optimal portfolios can be obtained through the solution of a single minimization, and that the resulting efficient frontiers are convex. We conclude with a real data example in which we compare optimal portfolios generated by a mean/Haezendonck criterion with mean/variance and mean/CVaR optimal portfolios.
\end{abstract}

\section{Introduction}

The aim of this work is to investigate several numerical issues concerning Haezendonck risk measures, as introduced in the papers of Haezendonck and Goovaerts [8] and of Goovaerts et al. [7], and recently studied in Bellini and Rosazza Gianin [4]. We start by sketching the definition and the basic theoretical properties of these measures, addressing an interested reader to Bellini and Rosazza Gianin [4] for a more detailed discussion.

We recall that the Haezendonck measure of a loss $X \in L^{\infty}$ is defined as

$$
\pi_{\alpha}(X)=\inf _{x \in \mathbb{R}}\left\{x+H_{\alpha}\left((X-x)^{+}\right)\right\}
$$

AMS 2000 classification: 60G42, 60G $44,62 \mathrm{P} 05,91 \mathrm{H} 30$

Key words and phrases: Haezendonck Measures, Orlicz Premiums, Coherent Risk Measures, Optimal Portfolios, Efficient Frontiers, Influence Functions. 
where $\alpha \in[0,1)$ and $H_{\alpha}$ is the so called Orlicz premium given by the (unique) solution of the equation

$$
E\left[\Phi\left(\frac{Y}{H_{\alpha}(Y)}\right)\right]=1-\alpha, \text { for } Y \in L_{+}^{\infty}, Y \neq 0,
$$

while $H_{\alpha}(0)=0$ by convention.

The function $\Phi:[0,+\infty) \rightarrow[0,+\infty)$ is sometimes called a (normalized) Young function and satisfies the following assumptions:

$$
\Phi(0)=0, \Phi(1)=1, \Phi(+\infty)=+\infty, \Phi \text { convex and strictly increasing, }
$$

see for instance Krasnosel'skii and Rutickii [12] and Rao and Ren [14] for the mathematical details.

The Orlicz premium $H_{\alpha}$ and consequently the Haezendonck measure $\pi_{\alpha}$ depend on the choice of the Young function $\Phi$ and of the parameter $\alpha$; in order to avoid heavy notations we will write simply $\pi_{\alpha}$ and $H_{\alpha}$ instead of $\pi_{\alpha}^{\Phi}$ and $H_{\alpha}^{\Phi}$ whenever no confusion may arise.

$>$ From a financial point of view, the function $\Phi$ may be interpreted as a loss function. When $\alpha=0$, the equation (1.2) then requires that the insurer is indifferent between the losses $\frac{Y}{H_{0}(Y)}$ and 1 ; this motivates the interpretation of the Orlicz premium as a version of the certainty equivalent that is positively homogeneous by construction.

When $\alpha \in(0,1)$, the parameter $\alpha$ is related to the degree of risk aversion of the insurer; it holds indeed that $H_{\alpha}(X)$ is strictly increasing in $\alpha \in[0,1)$ (see Bellini and Rosazza Gianin [4] for the proof). Although for general losses $Y$ the dependence of the Orlicz premium $H_{\alpha}(Y)$ on $\alpha$ is nonlinear, for constant $c \geq 0$ we have

$$
H_{\alpha}(c)=\frac{c}{\Phi^{-1}(1-\alpha)}
$$

that shows that the parameter $\alpha$ is linked in a one-to-one correspondence to the loading factor applied to sure losses.

The minimization construction that defines the Haezendonck measure $\pi_{\alpha}$ in definition (1.1) is similar to the optimized certainty equivalent introduced in Ben-Tal and Teboulle [5] and may have a similar financial interpretation. In a more actuarial fashion, we may see $(X-x)^{+}$as the payment for a loss $X$ when a franchise $x$ is applied; in this view the $x^{*}$ realizing the minimum corresponds to an optimal choice of the franchise from the point of view of the insurer.

$>$ From a mathematical point of view the functional $\pi_{\alpha}$ is translation invariant by construction and may be seen as an inf-convolution of two properly chosen convex functionals. Moreover, if $\alpha \neq 0$ the infimum in Definition 1.1 is always attained (see [4] for the proof), i.e. the inf-convolution is exact. For a recent general discussion on inf-convolutions and convex risk measures see for instance Barrieu and El Karoui [3].

A remarkable fact is that the Haezendonck measure associated to the Young function $\Phi(x)=x$ coincides with the Conditional Value at Risk (CVaR) as 
defined by Rockafellar and Uryasev [16], [17]. Hence the class of Haezendonck measures can be seen as a nonlinear generalization of the CVaR and actually does possess some of the nice computational features of the CVaR, as we will see in the following sections. The straightforward probabilistic interpretation of the parameter $\alpha$ and of the minimum point $x^{*}$, however, does not seem to hold in the more general situation of nonlinear $\Phi$. For a more detailed discussion and some relatively simple examples with power and exponential Young functions see again Bellini and Rosazza Gianin [4].

The most important theorical properties of the associated risk measure $\rho_{\alpha}(X) \triangleq \pi_{\alpha}(-X)$, that we call Haezendonck risk measure in order to avoid sign confusions, is its coherence in the sense of Artzner, Delbaen, Eber and Heath [2]. The crucial issue is its subadditivity for general losses that has been proved in Bellini and Rosazza Gianin [4], where also a characterization of the corresponding set of generalized scenarios has been provided.

For a practical use of Haezendonck risk measures, the first problem is the numerical computation of the Orlicz premium $H_{\alpha}$. In the next section we will study the properties of the natural estimator $\hat{H}_{\alpha}(X)$ that is defined as the unique solution of the equation

$$
\frac{1}{N} \sum_{i=1}^{N} \Phi\left(\frac{x_{i}}{\widehat{H}_{\alpha}(X)}\right)=1-\alpha,
$$

where $x_{i}, i=1, \ldots, N$, is a random sample of independent observations from the distribution $X$.

After a simulation experiment we will show that $H_{\alpha}(X)$ can be seen as an $M$ functional in the sense of Huber [11]; see also Serfling [18] for further details. This will enable us to derive the asymptotic consistency and the asymptotic normality of $\widehat{H}_{\alpha}(X)$ from general arguments. Moreover, we will compute in an explicit form the influence function (see for example Hampel [9]) of the functional $H_{\alpha}$ so that its dependence on the Young function $\Phi$ will become apparent.

Unfortunately, the same methodology does not applies to the Haezendonck measure $\pi_{\alpha}(X)$, for which the explicit computation of the influence function is quite awkward. The reason is that $\pi_{\alpha}(X)$ is not comonotone (with the trivial exception of the CVaR case) and hence cannot be represented as a convex combination of CVaR's related to different parameters $\alpha$, as in Kusuoka [13] or in Acerbi [1]. We will present only a simulation study of the distributional properties of

$$
\widehat{\pi}_{\alpha}(X)=\inf _{x \in \mathbb{R}} \widehat{\pi}_{\alpha}(X, x),
$$

where $\widehat{\pi}_{\alpha}(X, x)$ is defined as

$$
\widehat{\pi}_{\alpha}(X, x)=\widehat{H}_{\alpha}\left((X-x)^{+}\right)+x .
$$


The last part of the paper will be devoted to portfolio optimization problems with Haezendonck risk measures. We will first prove that as in the CVaR case (see Rockafellar and Uryasev [16], [17]) the optimal mean/Haezendonck portfolios can be obtained through a single minimization, and that the resulting efficient frontiers are convex (see for instance Bertsimas et al. [6]) for the proof in the CVaR case). We will conclude with a real data example based on the past four years returns of the first five stocks of the SPMIB Index in which we compare optimal portfolios and efficient frontiers obtained by using the CVaR, the variance, and two Haezendonck measures with Young functions $\Phi_{2}(x)=$ $\frac{x+x^{2}}{2}, \Phi_{3}(x)=\frac{e^{x}-1}{e-1}$.

The paper is organized as follows. In Section 2 we deal with the problem of the numerical computation of $H_{\alpha}$ and we derive the properties of $\widehat{H}_{\alpha}(X)$; in Section 3 we investigate the distribution of $\widehat{\pi}_{\alpha}$ and the joint distribution of $x^{*}$ and $\widehat{\pi}_{\alpha}$; Section 4 is devoted to portfolio optimization. The proofs of the results of Section 4 are postponed to the Appendix.

\section{Numerical computation of $H_{\alpha}$}

In this section we investigate the numerical computation of the Orlicz premium $H_{\alpha}$. We remind that an analytic expression for $H_{\alpha}(X)$ is available only for special cases of the Young function $\Phi$ and of the distribution of $X$, see Bellini and Rosazza Gianin [4].

We define the quantity $\widehat{H}_{\alpha}(X)$ as the natural sample equivalent of $H_{\alpha}(X)$, that is as the solution of the equation

$$
\frac{1}{N} \sum_{i=1}^{N} \Phi\left(\frac{x_{i}}{\widehat{H}_{\alpha}(X)}\right)=1-\alpha,
$$

where $x_{i}, i=1, \ldots, N$, is a random sample of independent observations with distribution $X$. From the general results in Haezendonck and Goovaerts [8] it follows that this equation has always exactly one solution for any $X \in L_{+}^{\infty}, X \neq$ 0 .

We start our analysis with a numerical experiment in which we analyze the distribution of $\widehat{H}_{\alpha}(X)$ on repeated simulated data for the Young function

$$
\Phi(x)=q x+(1-q) x^{2} .
$$

We remind that in this case the true value of the Orlicz premium $H_{\alpha}(X)$ can be calculated explicitly. When $\alpha=0$, its espression is the following:

$$
H_{0}(X)=\frac{q E[X]+\sqrt{q^{2} E^{2}[X]+4(1-q) E\left[X^{2}\right]}}{2} .
$$

The knowledge of the true value $H_{0}(X)$ will enable us to assess the unbiasedness of the empirical quantity $\widehat{H}_{0}(X)$. 
We will consider simulated data from the following three distributions:

- Uniform $(0,1)$

- Exponential with $\lambda=1$

- Pareto with $a=4$.

We remark that although the Exponential and the Pareto do not belong to $L^{\infty}$, they do have the first two moments finite, hence for the selected Young function the Orlicz premium is still finite and well defined (see Haezendonck and Goovaerts [8]).

The theorical values of the Orlicz premia $H_{0}(X)$ for the three considered distributions are reported in Table 2.1.

Table 2.1: Theorical value of the Orlicz Premia for the three considered distributions

\begin{tabular}{||l||l||l||l||}
\hline \hline & Uniform $(0, c)$ & Exponential $(\lambda)$ & Pareto $(a)$ \\
\hline \hline density & $f(x)=\frac{1}{c}$ if $x \in[0, c]$ & $f(x)=\lambda e^{-\lambda x}$ if $x \geq 0$ & $f(x)=a x^{-a-1}$ if $x \geq 1$ \\
\hline \hline$H_{0}(X)$ & $\frac{1}{2} c\left(\frac{1}{2} q+\sqrt{\frac{q^{2}}{4}+\frac{4}{3}(1-q)}\right)$ & $\frac{1}{\lambda}\left(\frac{q+\sqrt{q^{2}+8(1-q)}}{2}\right)$ & $\frac{a}{a-1} \frac{q+\sqrt{q^{2}+4(1-q) \frac{(a-1) 2}{a(a-2)}}}{2}$ \\
\hline \hline
\end{tabular}

The parameter values chosen for our numerical experiment are $c=1, \lambda=1$, $a=4, q=\frac{1}{2}$. For the three distributions above with these parameters the true values of $H_{0}(X)$ are reported in the first line of Table 2.2 .

The simulation experiment is designed as follows: for each of the three distributions considered, we simulate 1000 values and compute the corresponding value of $\widehat{H}_{0}(X)$ by solving numerically equation $(2.1)$. This is performed in Matlab environment by using the function fsolve. The procedure is then iterated 1000 times thus originating three distributions of 1000 values of $\widehat{H}_{0}(X)$, one for each of the three sampling distributions.

In Table 2.2 we compare the average values of $\widehat{H}_{0}(X)$ with their theorical values $H_{0}(X)$ and report some other relevant statistics of the distribution of $\widehat{H}_{0}(X)$.

The results does not show any systematic bias of $\widehat{H}_{0}(X)$. The distributions of $\widehat{H}_{0}(X)$ in the three cases are plotted in Figure 5.1.

\section{Insert Fig. 1 about here}

From a graphical point of view, we see that the distributions of $\widehat{H}_{0}(X)$ are unimodal and roughly symmetric in the Uniform and Exponential case, while in the Pareto case it is asymmetric and heavy-tailed. A standard Jarque-Bera test does not reject the null hypothesis of normality at the $1 \%$ level for the first two cases, while it is rejected at $5 \%$ level in the Pareto case. We will see in the next section that the asymptotic unbiasedness can be proved under 
Table 2.2: Basic statistics of the distributions of $\widehat{H}_{0}(X)$

\begin{tabular}{||l||l||l||l||}
\hline \hline & Uniform & Exponential & Pareto \\
\hline \hline$H_{0}(X)$ & 0.55196 & 1.2808 & 1.3874 \\
\hline \hline$E\left[\widehat{H}_{0}\right]$ & 0.5521 & 1.2806 & 1.3862 \\
\hline \hline Std $\left[\widehat{H}_{0}\right]$ & 0.0084 & 0.0425 & 0.0280 \\
\hline \hline Skew $\left[\widehat{H}_{0}\right]$ & -0.0292 & 0.0833 & 1.6618 \\
\hline \hline Kurt $\left[\widehat{H}_{0}\right]$ & 2.9569 & 2.9755 & 10.3411 \\
\hline \hline
\end{tabular}

very general conditions and actually holds in the three considered examples. Asymptotic normality holds under additional assumptions that actually for our choice of $\Phi$ does not hold in the Pareto case, in accordance with the simulation results.

\subsection{Orlicz premiums as M-functionals: asymptotic consis- tency and asymptotic normality of $\widehat{H}_{0}$}

The empirical results obtained in the previous section can be derived from the asymptotic properties of the so called $M$-functionals (see Huber [11] or Serfling [18] for a detailed treatment). We restate here for completeness the basic definitions, focusing on the case $\alpha=0$ for notational simplicity.

Let us denote with $F$ the distribution function of $X$. An $M$-functional is a functional $H(F)$ defined as the solution of the equation

$$
\int \psi(x, H(F)) d F(x)=0,
$$

where $\psi(x, t)$ is a given function $\psi: \mathbb{R}^{2} \rightarrow \mathbb{R}$.

It is clear that the Orlicz premiums are $M$-functionals with

$$
\psi(x, t)=\Phi\left(\frac{x}{t}\right)-1
$$

for $\alpha=0$ and with

$$
\psi_{\alpha}(x, t)=\Phi\left(\frac{x}{t}\right)-(1-\alpha)
$$

for $\alpha \in(0,1)$.

Denote by $F_{n}$ the empirical distribution function of a sample of $n$ independent observations drawn from $F$ and denote by $\widehat{H}_{n}$ the solution of the equation

$$
\int \psi\left(x, \widehat{H}_{n}\left(F_{n}\right)\right) d F_{n}(x)=0 .
$$

The asymptotic consistency of $M$-functionals can be proved under very mild hypothesis (see Serfling [18], p. 249): 
"If $H(F)$ is an isolated root of the equation

$$
\int \psi(x, H(F)) d F(x)=0
$$

and if $\psi(x, t)$ is monotone in $t$, then $H(F)$ is unique and any solution sequence $\widehat{H}_{n}$ converges to $H$ almost surely".

In the Orlicz case the solution $H(F)$ of the equation that defines the premium is always unique. The solutions of the sample equations $\widehat{H}_{n}$ always exist and are unique. The function $\psi(x, t)=\Phi\left(\frac{x}{t}\right)-1$ is clearly monotone in $t$, hence we have asymptotic consistency of the Orlicz premia $\widehat{H}_{n}$, that is

$$
\widehat{H}_{n}\left(F_{n}\right) \rightarrow H(F) \text { a.s. for } n \rightarrow+\infty \text {. }
$$

For what concerns asymptotic normality, we have the following result (see Serfling [18], p. 251):

"Let $H(F)$ be an isolated root of the equation

$$
\int \psi(x, H(F)) d F(x)=0
$$

Let $\psi(x, t)$ be monotone in $t$; let

$$
\lambda_{F}(t)=\int \psi(x, t) d F(x)
$$

if

$$
\lambda_{F}^{\prime}(H(F)) \neq 0
$$

and

$$
\int \psi^{2}(x, t) d F(x)<+\infty
$$

in a neighborhood of $H(F)$ and it is continuous in $H(F)$, then $\widehat{H}_{n}$ is asymptotically normal, that is

$$
\sqrt{n}\left(\widehat{H}_{n}-H\right) \rightarrow^{d} N\left(0, \sigma^{2}(H, F)\right)
$$

where

$$
\sigma^{2}(H, F)=\frac{\int \psi^{2}(x, H(F)) d F(x)}{\left[\lambda_{F}^{\prime}(H(F))\right]^{2}}
$$

Note that when the conditions (2.7) and (2.8) are satisfied and when it is possible to exchange the derivative with respect to $t$ and the expectation, (2.10) can be rewritten as

$$
\sigma^{2}(H, F)=\frac{H(X)\left[1-2 E\left[\Phi\left(\frac{X}{H(X)}\right)\right]-E\left[\Phi^{2}\left(\frac{X}{H(X)}\right)\right]\right]}{E\left[\frac{X}{H(X)} \Phi^{\prime}\left(\frac{X}{H(X)}\right)\right]}
$$


In the Orlicz case, condition (2.8) is equivalent to

$$
E\left[\Phi^{2}\left(\frac{X}{t}\right)\right]<+\infty
$$

that is always satisfied if $X$ belongs to $L^{\infty}$, while it has to be checked explicitly for more general $X$.

To be more concrete, consider once more the function $\Phi$ in (2.2) with $q=\frac{1}{2}$. It is immediate to check that condition (2.7) is always satisfied. Furthermore, in our three examples we have that condition (2.11) is satisfied in the Uniform and in the Exponential case, while it is not satisfied in the Pareto case, since with $a=4$ the fourth moment is not finite. Hence, in this case the limit distribution may be not normal, as it is confirmed by the numerical simulations.

In the Uniform and Exponential cases it is also possible to compute explicitly the asymptotic variance by means of expression (2.10). We have indeed that

$$
\psi(x, t)=\frac{x}{2 t}+\frac{x^{2}}{2 t^{2}}-1 .
$$

In the Uniform case, we have

$$
\lambda_{F}(t)=\int \psi(x, t) d F(x)=\frac{1}{4 t}+\frac{1}{6 t^{2}}-1
$$

and

$$
\int \psi^{2}(x, t) d F(x)=\frac{1}{8 t^{3}}-\frac{1}{4 t^{2}}-\frac{1}{2 t}+\frac{1}{20 t^{4}}+1,
$$

hence

$$
\sigma^{2}(H, F)=\left.\frac{\frac{1}{8 t^{3}}-\frac{1}{4 t^{2}}-\frac{1}{2 t}+\frac{1}{20 t^{4}}+1}{\frac{1}{16 t^{4}}+\frac{1}{6 t^{5}}+\frac{1}{9 t^{6}}}\right|_{t=H_{0}(X)} .
$$

In the Exponential case, we have

$$
\lambda_{F}(t)=\int \psi(x, t) d F(x)=\frac{1}{2 t}+\frac{1}{t^{2}}-1
$$

and

$$
\int \psi^{2}(x, t) d F(x)=\frac{3}{t^{3}}-\frac{3}{2 t^{2}}-\frac{1}{t}+\frac{6}{t^{4}}+1
$$

hence

$$
\sigma^{2}(H, F)=\left.\frac{\frac{3}{t^{3}}-\frac{3}{2 t^{2}}-\frac{1}{t}+\frac{6}{t^{4}}+1}{\frac{1}{4 t^{4}}+\frac{2}{t^{5}}+\frac{4}{t^{6}}}\right|_{t=H_{0}(X)} .
$$

With the chosen parameters we get as theorical standard deviations of $\widehat{H}_{0}$ the values

$$
\frac{\sigma\left(\widehat{H}_{0}\right)_{\text {Uniform }}}{\sqrt{1000}}=0.00841 ; \quad \frac{\sigma\left(\widehat{H}_{0}\right)_{\text {Exponential }}}{\sqrt{1000}}=0.0433
$$

that are in good accordance with the empirical values presented in Table 2.2. 


\subsection{The influence function of $\widehat{H}_{0}$}

The connection that we have established between Orlicz premia and $M$-functionals makes possible also to compute explicitly their influence function. Again we recall for completeness the basic definitions and results (see Hampel [9] and Serfling [18] for further details). The Gateaux differential of a generic real functional $T$ defined on a space of probability distributions is defined as:

$$
d_{1} T(F, G-F) \triangleq \lim _{\lambda \rightarrow 0^{+}} \frac{T((1-\lambda) F+\lambda G)-T(F))}{\lambda}
$$

provided that the limit exists, and it is simply the "directional derivative" of the functional $T$ evaluated in the point $F$ along the direction of the distribution $G$.

For $M$-functionals the Gateaux differential can be computed as follows (see Serfling [18], p. 245):

$$
d_{1} T(F, G-F)=-\frac{\int \psi(x, T(F)) d G(x)}{\lambda_{F}^{\prime}(T(F))} .
$$

A very important special case of Gateaux differential is when the distribution $G$ is concentrated in one point $x$, that is $G=\delta_{x}$. In this case the Gateaux differential, known as the influence function of $T$ (see Hampel [9] for further details),

$$
I C(x, F, T)=\lim _{\lambda \rightarrow 0^{+}} \frac{\left.T\left((1-\lambda) F+\lambda \delta_{x}\right)-T(F)\right)}{\lambda}
$$

represents the infinitesimal variation of $T$ due to a "contamination" of the original distribution $F$ with the addition of mass in the point $x$.

For a general $M$-functional its influence function is given by

$$
I C(x, F, T)=-\frac{\psi(x, T(F))}{\lambda_{F}^{\prime}(T(F))},
$$

hence the influence function of the Orlicz premium $H_{0}$ is given by

$$
I C(x, F, H)=\frac{1-\Phi\left(\frac{x}{H(X)}\right)}{\left.\frac{d}{d t} E\left[\Phi\left(\frac{X}{t}\right)\right]\right|_{t=H(X)}} .
$$

Note that when it is possible to exchange the derivative with respect to $t$ and the expectation, (2.12) can be rewritten as

$$
I C(x, F, H)=\frac{H(X)\left[\Phi\left(\frac{x}{H(X)}\right)-1\right]}{E\left[\frac{X}{H(X)} \Phi^{\prime}\left(\frac{X}{H(X)}\right)\right]} .
$$

For a fixed $X$, the asymptotic behaviour of $I C(x, F, H)$ for $x \rightarrow+\infty$ is the same as $\Phi(x)$, up to a multiplicative and to an additive constant. This shows 
how the choice of $\Phi$ impacts on the corresponding $H_{0}(X)$ : the faster $\Phi$ goes to infinity, the more sensible the premium $H_{0}$ is to a perturbation in the tail of the distribution.

Typically, Young functions grows like powers or exponentials (with the exception of the CVaR case for which $\Phi(x)=x$ ), thus indicating a remarkable sensitivity to a "contamination" in the tails. In robust statistics this would be considered extremely undesirable, since it would imply a huge variation of the considered functional in presence of an outlier (that could be simply an erroneous point in the dataset).

In financial practice, however, extremal values of the underlying stocks or indices are quite common and contribute to the final performance exactly as "normal" data; they can hardly be interpreted as the result of a reporting error. Hence, we consider the influence function as a descriptive tool that captures some characteristics of the chosen risk measure rather than a normative tool that discriminates between robust risk measures (that is with bounded influence function) and non-robust risk measures (that is with unbounded influence function).

\section{$3 \quad$ Numerical computation of $x^{*}$ and of $\pi_{\alpha}(X)$}

The theorical results of the previous section give us a considerable amount of information about the asymptotic properties of $\widehat{H}_{\alpha}(X)$. We now move to the problem of the analysis of

$$
\widehat{\pi}_{\alpha}(X)=\inf _{x \in \mathbb{R}} \widehat{\pi}_{\alpha}(X, x),
$$

where

$$
\widehat{\pi}_{\alpha}(X, x)=\widehat{H}_{\alpha}\left((X-x)^{+}\right)+x,
$$

that is the empirical version of the Haezendonck measure $\pi_{\alpha}(X)$.

$>$ From our previous work (see [4]) we know that the infimum in definition (3.1) is always attained if $\alpha \neq 0$; we will denote the minimum point with $\widehat{x}^{*}$.

In comparison with the analysis carried on in the previous section on $\widehat{H}_{\alpha}$, in this case the situation is much less clear. We are neither able to derive in an analytic fashion the influence function of $\widehat{\pi}_{\alpha}(X)$ nor to prove its asymptotic consistency, since in general the functional $\pi_{\alpha}(X)$ is not comonotone, hence it cannot be represented as an integral combination of CVaR's as in the Kusuoka's general representation (see Kusuoka [13]). It follows that the Haezendonck measure is not in general a "spectral measure" in the terminology of Acerbi [1], hence its influence function cannot be computed in a straightforward fashion as an integral of influence functions of CVaR's with different $\alpha$. For this reason we limit ourselves to a simulation study of the distributions of $\widehat{x}^{*}$ and $\widehat{\pi}_{\alpha}(X)$.

First of all, we show an example of the computation of $\widehat{\pi}_{\alpha}(X)$ on simulated data from the three distributions previously considered and from a standard normal distribution. We compute numerically the quantities (3.2) and minimize 
them as in the definition (3.1) on a sample of $N=1000$ values generated from the four aforementioned distributions. The minimization is performed in Matlab environment by means of the fmincon function. The procedure is repeated for the Young functions:

$$
\Phi_{1}(x)=x, \quad \Phi_{2}(x)=\frac{x+x^{2}}{2}, \quad \Phi_{3}(x)=\frac{e^{x}-1}{e-1}
$$

and when the parameter $\alpha$ is set to 0.95 .

The values of $x^{*}$ and $\widehat{\pi}_{0.95}(X)$ for the 12 different cases (3 Young functions $\mathrm{x} 4$ sampling distributions) are reported in Table 3.1. Since for $\Phi_{1}(x)=x$ the theoretical minimum point $x^{*}$ coincides with the 95 -percentile, we report it in the last line of Table 3.1 for comparison.

Table 3.1: Example of computation of $\widehat{x}^{*}$ and $\widehat{\pi}_{0.95}(X)$

\begin{tabular}{||c||c||c||c||c||c||}
\hline \hline & & Uniform & Exponential & Pareto & Normal \\
\hline \hline \multirow{2}{*}{$\Phi_{1}(x)=x$} & $\widehat{x}^{*}$ & 0.9495 & 3.0509 & 2.2630 & 1.4985 \\
& $\widehat{\pi}_{0.95}(X)$ & 0.9716 & 4.1730 & 3.0685 & 1.8651 \\
\hline \hline \multirow{2}{*}{$\Phi_{2}(x)=\frac{x+x^{2}}{2}$} & $\widehat{x}^{*}$ & 0.9424 & 2.7090 & 2.0628 & 1.3770 \\
& $\widehat{\pi}_{0.95}(X)$ & 0.9742 & 4.5068 & 3.2481 & 1.9394 \\
\hline \hline \multirow{2}{*}{$\Phi_{3}(x)=\frac{e^{x}-1}{e-1}$} & $\widehat{x}^{*}$ & 0.9411 & 2.4699 & 1.9798 & 1.3395 \\
& $\widehat{\pi}_{0.95}(X)$ & 0.9752 & 4.8203 & 3.3429 & 1.9770 \\
\hline \hline 95 -percentile & - & 0.9495 & 3.0509 & 2.2630 & 1.4985 \\
\hline \hline
\end{tabular}

We see that $\widehat{x}^{*}$ is always lower than the corresponding $\widehat{\pi}_{0.95}(X)=\widehat{\pi}_{0.95}\left(X, \widehat{x}^{*}\right)$; this is clear in the $\Phi_{1}$ case from the probabilistic interpretation of the CVaR, and it is true in general for the Haezendonck measures from the inequality $\pi_{\alpha}(X, x) \geq x$, although it is not clear if a similar probabilistic interpretation of $\pi_{\alpha}(X)$ may be provided.

Moreover, comparing the Haezendonck measures $\widehat{\pi}_{0.95}(X)$ for the three different Young functions, we see that for all distributions they increase moving from $\Phi_{1}$ to $\Phi_{2}$ and $\Phi_{3}$. We could say roughly that these three Young functions are ordered according to increasing risk-aversion, although they do not satisfy the ordering condition proposed in Haezendonck and Goovaerts [8]. Anyway, it is always true (as a consequence of the well known properties of $H_{0}$ ) that at least for $\alpha=0$ it holds $\pi_{0}^{\Phi_{1}} \leq \pi_{0}^{\Phi}$ for any Young function satisfying the hypothesis in (1.3).

The functions $\widehat{\pi}_{0.95}(X, x)$ in the 12 considered cases are plotted in Fig. 5.2.

\section{Insert Fig. 2 about here}

As it is clear from the definitions, in all cases we have that $\widehat{\pi}_{0.95}(X, x) \sim x$ for $x \rightarrow+\infty$; moreover, as proved in [4], we see that the functions $\widehat{\pi}_{0.95}(X, x)$ are strictly convex and have exactly one global minimum point $\widehat{x}^{*}$. 
In order to analyze the distributional properties of $\widehat{x}^{*}$ and $\widehat{\pi}_{0.95}\left(X, \widehat{x}^{*}\right)$ we repeat 500 times the preceding experiment and in each case we determine the corresponding couple $\left(\widehat{x}^{*}, \widehat{\pi}_{0.95}\left(X, \widehat{x}^{*}\right)\right)$. We thus produce 12 simulated distributions of 500 couples $\left(\widehat{x}^{*}, \widehat{\pi}_{0.95}\left(X, \widehat{x}^{*}\right)\right)$. In Tables 3.2 and 3.3 we analyze separately the marginals distributions of $\widehat{x}^{*}$ and $\widehat{\pi}_{0.95}\left(X, \widehat{x}^{*}\right)$.

Table 3.2: Basic statistics of the distribution of $\widehat{x}^{*}$ in the 12 considered cases

\begin{tabular}{|l|c|c|c|c|c|}
\hline & & Uniform & Exponential & Pareto & Normal \\
\hline \multirow{3}{*}{$\Phi_{1}(x)=x$} & 95 -percentile & 0.9500 & 2.9957 & 2.1147 & 1.6449 \\
& $E\left(\widehat{x}^{*}\right)$ & 0.9497 & 2.9967 & 2.1173 & 1.6426 \\
& Std $\left(\widehat{x}^{*}\right)$ & 0.0067 & 0.1398 & 0.0736 & 0.0619 \\
& $J B p-$ value & $\mathbf{0 . 0 1 5 7}$ & 0.3552 & 0.1450 & 0.7563 \\
\hline \multirow{3}{*}{$\Phi_{2}(x)=\frac{x+x^{2}}{2}$} & $E\left(\widehat{x}^{*}\right)$ & 0.9424 & 2.6782 & 1.9034 & 1.5161 \\
& Std $\left(\widehat{x}^{*}\right)$ & 0.0075 & 0.1172 & 0.0716 & 0.0599 \\
& $J B p-$ value & $\mathbf{0 . 0 0 8 5}$ & $<\mathbf{1 0}^{-3}$ & 0.4056 & 0.0862 \\
\hline \multirow{2}{*}{$\Phi_{3}(x)=\frac{e^{x}-1}{e-1}$} & $E\left(\widehat{x}^{*}\right)$ & 0.9410 & 2.5430 & 1.7774 & 1.4538 \\
& Std $\left(\widehat{x}^{*}\right)$ & 0.0075 & 0.1383 & 0.1019 & 0.0647 \\
& $J B p-$ value & 0.4404 & $\mathbf{0 . 0 1 3 8}$ & $<\mathbf{1 0}^{-3}$ & 0.5287 \\
\hline
\end{tabular}

Table 3.3: Basic statistics of the distributions of $\widehat{\pi}_{0.95}(X)$ in the 12 considered cases

\begin{tabular}{|l|c|c|c|c|c|}
\hline & & Uniform & Exponential & Pareto & Normal \\
\hline \multirow{3}{*}{$\Phi_{1}(x)=x$} & $C V a R_{0.95}(X)$ & 0.9750 & 3.9958 & 2.8198 & 2.0626 \\
& $E\left(\widehat{\pi}_{0.95}(X)\right)$ & 0.9745 & 3.9842 & 2.8222 & 2.0536 \\
& $S t d\left(\widehat{\pi}_{0.95}(X)\right)$ & 0.0040 & 0.2040 & 0.1730 & 0.0748 \\
& $J B p-$ value & $<\mathbf{1 0}^{-3}$ & 0.0979 & $<\mathbf{1 0}^{-3}$ & 0.1316 \\
\hline \multirow{3}{*}{$\Phi_{2}(x)=\frac{x+x^{2}}{2}$} & $E\left(\widehat{\pi}_{0.95}(X)\right)$ & 0.9770 & 4.2205 & 3.0952 & 2.1423 \\
& $S t d\left(\widehat{\pi}_{0.95}(X)\right)$ & 0.0036 & 0.2333 & 0.3203 & 0.0869 \\
& $J B p-$ value & $\mathbf{0 . 0 0 6 7}$ & 0.2169 & $<\mathbf{1 0}^{-3}$ & 0.2073 \\
\hline \multirow{3}{*}{$\Phi_{3}(x)=\frac{e^{x}-1}{e-1}$} & $E\left(\widehat{\pi}_{0.95}(X)\right)$ & 0.9776 & 4.3865 & 3.4064 & 2.1876 \\
& $S t d\left(\widehat{\pi}_{0.95}(X)\right)$ & 0.0037 & 0.2649 & 0.6507 & 0.0929 \\
& $J B p-$ value & 0.1202 & $<\mathbf{1 0}^{-3}$ & $<\mathbf{1 0}^{-3}$ & 0.8674 \\
\hline
\end{tabular}

In the first line of Table 3.2 we report the theorical values of $x^{*}$ while in the first line of Table 3.3 we report the theorical values of $C V a R_{0.95}(X)$. The results do not show any systematic bias in both cases, although from a distributional point of view the situation is much more complex than in the $\widehat{H}_{\alpha}(X)$ case. Indeed, we see that the Jarque-Bera test rejects normality at $5 \%$ level in 5 over 
12 cases for what concerns $\widehat{x}^{*}$ and in 6 over 12 cases for what concerns $\widehat{\pi}_{0.95}(X)$ (the cases where the normality is rejected are emphasized in bold).

The reason for this apparently erratic behaviour is that these quantities are affected both by the numerical error in the computation of $\widehat{H}_{\alpha}(X)$ and by the numerical error in the minimization of $\widehat{\pi}_{\alpha}(X, x)$; this means that the simulation analysis of the distributions of $\widehat{x}^{*}$ and $\widehat{\pi}_{\alpha}\left(X, \widehat{x}^{*}\right)$ is quite difficult to perform and would require an accurate control of the numerical minimization algorithm. For this reason we just present in Figure 5.3 the joint distribution of $\left(\widehat{x}^{*}, \widehat{\pi}_{0.95}\left(X, \widehat{x}^{*}\right)\right)$.

Insert Fig. 3 about here

It is interesting to note that the two quantities $\widehat{x}^{*}$ and $\widehat{\pi}_{0.95}(X)$ are often correlated; the error on $\widehat{x}^{*}$ it is clearly not independent from the error on $\widehat{\pi}_{0.95}(X)$. However, contrary to the intuition this correlation is not necessarily always positive, as reported in Table 3.4.

Table 3.4: Empirical correlations between $\widehat{x}^{*}$ and $\widehat{\pi}_{0.95}(X)$

\begin{tabular}{||l||l||l||l||l||}
\hline \hline & Uniform & Exponential & Pareto & Normal \\
\hline \hline$\Phi_{1}(x)=x$ & 0.8479 & 0.7276 & 0.5809 & 0.7189 \\
\hline \hline$\Phi_{2}(x)=\frac{x+x^{2}}{2}$ & 0.8548 & 0.2771 & -0.3058 & 0.5076 \\
\hline \hline$\Phi_{3}(x)=\frac{e^{x}-1}{e-1}$ & 0.8692 & -0.0040 & -0.6734 & 0.3631 \\
\hline \hline
\end{tabular}

\section{Optimal portfolios}

In this section we deal with the problem of computing optimal portfolios in a mean/Haezendonck setting and we compare them with mean/variance and mean/CVaR portfolios in a real data example.

The determination of optimal portfolios with respect to the Haezendonck risk measure $\rho_{\alpha}(X)$ actually involves two nested minimizations. Denoting by $\mathbf{X}=$ $\left(X_{1}, \ldots, X_{n}\right)$ the random vector of the assets' returns, by $\mathbf{r}=E[\mathbf{X}]$ their expected values, by $\mathbf{w}=\left(w_{1}, \ldots, w_{n}\right)$ the portfolio's weights and by $r_{p}$ the portfolio's target expected return, the optimization problem may be written as

$$
\min _{\mathbf{w} \in W, \mathbf{w} \cdot \mathbf{r}=r_{p}}\left\{\min _{x}\left\{\widehat{H}_{\alpha}\left((-\mathbf{X} \cdot \mathbf{w}-x)^{+}\right)+x\right\}\right\},
$$

where

$$
W \triangleq\left\{\mathbf{w} \in \mathbb{R}^{n}: w_{i} \geq 0 \text { and } \mathbf{w} \cdot \mathbf{1}=1\right\} .
$$

In (4.1), however, the two minimizations over $\mathbf{w}$ and over $x$ can be carried on in one single step, exactly as in the CVaR case. In other words a result similar to 
Theorem 14 of Rockafellar and Uryasev [17] holds for Haezendonck measures, that implies that the minimization problem in (4.1) is equivalent to the problem

$$
\min _{\mathbf{w} \in W, \mathbf{w} \cdot \mathbf{r}=r_{p}, x \in \mathbb{R}}\left\{\widehat{H}_{\alpha}\left((-\mathbf{X} \cdot \mathbf{w}-x)^{+}\right)+x\right\} .
$$

This is very important in practical applications since it reduces the computational complexity of the problem. We give below a formal statement, while the proof can be found in the Appendix.

Let $\mathbf{X} \in L^{\infty}$ and set

$$
\mathcal{X} \triangleq\{\mathbf{X} \cdot \mathbf{w} \text { for some } \mathbf{w} \in W\} .
$$

The following result holds true for any Haezendonck measure.

Proposition 4.1 For any $\alpha \in(0,1)$ :

$$
\begin{aligned}
& \text { (a) } \min _{X \in \mathcal{X}} \pi_{\alpha}(X)=\min _{(x, X) \in \mathbb{R} \times \mathcal{X}}\left\{x+H_{\alpha}\left((X-x)^{+}\right)\right\} . \\
& \text {(b) }\left(x^{*}, X^{*}\right) \in \arg \min _{(x, X) \in \mathbb{R} \times \mathcal{X}}\left\{x+H_{\alpha}\left((X-x)^{+}\right)\right\} \text {if and only if both } \\
& X^{*} \in \arg \min _{X \in \mathcal{X}} \pi_{\alpha}(X) \text { and } x^{*} \in \arg \min _{x \in \mathbb{R}}\left\{x+H_{\alpha}\left(\left(X^{*}-x\right)^{+}\right)\right\} \text {hold } \\
& \text { true. }
\end{aligned}
$$

$>$ From the result above and from the convexity of the functional $\pi_{\alpha}(X)$ it follows that in the $\alpha \neq 0$ case the solution of the minimization problems (4.1) or (4.2) always exist. In order to stress its dependence on $r_{p}$, we will denote by $\mathbf{w}^{*}\left(r_{p}\right)$ the optimal portfolio, by $X^{*}\left(r_{p}\right)$ the distribution of its return and by $\pi^{*}\left(r_{p}\right)=\pi_{\alpha}\left(X^{*}\left(r_{p}\right)\right)$ its Haezendonck measure. As in the Markowitz setting, the set of points $\left(r_{p}, \pi^{*}\left(r_{p}\right)\right)$ will be named the efficient frontier with respect to the mean/Haezendonck criterion.

The following result (whose proof can be found in the Appendix) is a straightforward generalization of the mean/CVaR case that has been proved in Bertsimas et al. [6].

Proposition 4.2 The efficient frontier is a convex curve in the plane $\left(r, \pi_{\alpha}\right)$.

We finally move to a portfolio example. Our aim is to compare the optimal portfolios obtained with three different Young functions with the mean/variance portfolio. We considered approximately 4 years of logreturns (1000 daily data) of the 5 main components of the SPMIB Index (that are Unicredito, Eni, Generali, Enel, Telecom) ranging from $14 / 11 / 2003$ to $18 / 10 / 2007$. The basic statistics of such logreturns are reported in Table 4.1.

The marginal distributions of the data show a strong departure from normality, as indicated by the values of the coefficients of skewness and kurtosis. The ranking of the riskiness of the different asset is the same with the standard deviation, the $95 \%$ - VaR and the $95 \%$-CVaR, although the ratios between these quantities are not constant, as they would be in the multivariate normal 
Table 4.1: Basic statistics of daily logreturns.

\begin{tabular}{|l|l|l|l|l|l|}
\hline & Unicredito & Eni & Intesa & Generali & Enel \\
\hline mean $\left(\mathrm{x} 10^{-4}\right)$ & 3.18 & 6.14 & 5.39 & 4.81 & 4.66 \\
\hline standard deviation (\%) & 1.18 & 1.06 & 1.36 & 1.01 & 0.92 \\
\hline skewness & -0.267 & -0.297 & -0.058 & 0.125 & -0.543 \\
\hline kurtosis & 5.718 & 3.850 & 5.924 & 6.018 & 6.402 \\
\hline $95 \%$ - VaR & -0.018 & -0.016 & -0.019 & -0.015 & -0.014 \\
\hline $95 \%$ - CVaR & -0.027 & -0.024 & -0.029 & -0.022 & -0.021 \\
\hline $\min (\%)$ & -0.062 & -0.044 & -0.071 & -0.045 & -0.058 \\
\hline $\max (\%)$ & 0.048 & 0.033 & 0.073 & 0.067 & 0.032 \\
\hline
\end{tabular}

Table 4.2: Correlation matrix of daily logreturns.

\begin{tabular}{|l|l|l|l|l|l|}
\hline & Unicredito & Eni & Intesa & Generali & Enel \\
\hline Unicredito & 1 & 0.383 & 0.472 & 0.506 & 0.294 \\
\hline Eni & 0.383 & 1 & 0.282 & 0.392 & 0.438 \\
\hline Intesa & 0.472 & 0.282 & 1 & 0.385 & 0.245 \\
\hline Generali & 0.506 & 0.392 & 0.385 & 1 & 0.340 \\
\hline Enel & 0.294 & 0.438 & 0.245 & 0.340 & 1 \\
\hline
\end{tabular}

case. The correlation matrix of the logreturns over the whole 4 -years period is displayed in Table 4.2.

We assume that short sales are not allowed and we compute the optimal portfolios for 50 different equally spaced values of the expected returns, both in the Markowitz case and for the Haezendonck risk measures related to the following three Young functions: $\Phi_{1}(x)=x$ (fi1), $\Phi_{2}(x)=\frac{x}{2}+\frac{x^{2}}{2}$ (fi2), $\Phi_{3}(x)=$ $\frac{e^{x}-1}{e-1}$ (fi3). Optimization has been performed in Matlab environment using the fmincon subroutine. To reduce numerical instabilities, various initialization points have been considered, including the optimal Markowitz portfolio and the optimal portfolio computed with the same $\Phi$ and with the closest expected return. With this procedure all the considered optimization did converge to an acceptable minimum.

The efficient frontiers are plotted in Figure 5.4.

Insert Fig. 4 about here

As expected, the shape of the different frontiers does not change much at least from a qualitative point of view in the various cases. The slight departure from convexity that is observed occasionally is due to the numerical approximations of the optimization algorithm. In order to better display the difference between the various optimality criteria, in Figure 5.5 we plot the weights of the optimal 
portfolios as a function of the target expected return $r_{p}$.

\section{Insert Fig.5 about here}

As it is well known, in the Markowitz case these weights are a linear function of the expected return; this is confirmed in the upper left part of Fig. 5.5. We see that the optimal weights for Haezendonck measures are quite different from Markowitz weights, although they show a similar dependence, at least in the bounds given by computational approximations. This difference is a direct consequence of the departure from multivariate normality of the returns' distribution; otherwise it is well known that all coherent risk measures would produce the same optimal portfolios.

Summing up, we can conclude from these preliminary numerical examples that the numerical optimization of Haezendonck risk measures can be implemented. It must be remarked that computational times are significantly greater than in the CVaR case, since the problem in general does not reduce to a linear programming problem. The experiment was run in Matlab environment by using built-in optimization routines such as fmincon; the typical time needed is around 10 secs for each point of the frontier.

Acknowledgements. The authors wish to thank Professor Pflug, Professor Rockafellar and all the participants of the Workshop "Models of Credit and Operational Risks in the Financial Sector "(held in Bolzano in September 2007) whose suggestions and comments have contributed to improve this paper.

\section{Appendix}

Proof of Proposition 4.1. (a) Set $f\left(w_{1}, \ldots, w_{n}\right) \triangleq \pi_{\alpha}\left(\sum_{i=1}^{n} w_{i} X_{i}\right)$ for $\left(w_{1}, \ldots, w_{n}\right) \in \mathbb{R}^{n}$. Since $f$ is convex in $\left(w_{1}, \ldots, w_{n}\right) \in \mathbb{R}^{n}$ (hence also continuoussee Rockafellar [15]), the infimum of $f$ over $W$ is attained. Hence:

$$
\min _{X \in \mathcal{X}} \pi_{\alpha}(X)=\min _{\left(w_{1}, \ldots, w_{n}\right) \in W} \pi_{\alpha}\left(\sum_{i=1}^{n} w_{i} X_{i}\right) .
$$

By definition of $\pi_{\alpha}$ and since in that definition the infimum is always attained for $\alpha \in(0,1)$ (see Proposition 16 of Bellini and Rosazza Gianin [4]), we deduce that

$$
\min _{X \in \mathcal{X}} \pi_{\alpha}(X)=\min _{X \in \mathcal{X}} \min _{x \in \mathbb{R}} \pi_{\alpha}(X, x)=\min _{(x, X) \in \mathbb{R} \times \mathcal{X}}\left\{x+H_{\alpha}\left((X-x)^{+}\right)\right\} .
$$

(b) On one hand, suppose that $\left(x^{*}, X^{*}\right) \in \arg \min _{(x, X) \in \mathbb{R} \times \mathcal{X}}\left\{x+H_{\alpha}\left((X-x)^{+}\right)\right\}$ and set $f\left(x^{*}, X^{*}\right) \triangleq x^{*}+H_{\alpha}\left(\left(X^{*}-x^{*}\right)^{+}\right)$. Hence

$$
\begin{aligned}
\pi_{\alpha}\left(X^{*}\right) & =f\left(x^{*}, X^{*}\right) \leq \min _{x \in \mathbb{R}}\left\{x+H_{\alpha}\left((X-x)^{+}\right)\right\}, \forall X \in \mathcal{X} \\
f\left(x^{*}, X^{*}\right) & =\pi_{\alpha}\left(X^{*}\right)=\min _{x \in \mathbb{R}}\left\{x+H_{\alpha}\left(\left(X^{*}-x\right)^{+}\right)\right\} .
\end{aligned}
$$


From (5.1) it follows that $X^{*} \in \arg \min _{X \in \mathcal{X}} \pi_{\alpha}(X)$, while, given such a $X^{*}$, from (5.2) it follows that $x^{*} \in \arg \min _{x \in \mathbb{R}}\left\{x+H_{\alpha}\left(\left(X^{*}-x\right)^{+}\right)\right\}$.

On the other hand, suppose that $X^{*} \in \arg \min _{X \in \mathcal{X}} \pi_{\alpha}(X)$ and $x^{*} \in$ $\arg \min _{x \in \mathbb{R}}\left\{x+H_{\alpha}\left(\left(X^{*}-x\right)^{+}\right)\right\}$. Hence

$$
\min _{X \in \mathcal{X}} \pi_{\alpha}(X)=\pi_{\alpha}\left(X^{*}\right)=\min _{x \in \mathbb{R}} \pi_{\alpha}\left(X^{*}, x\right)=x^{*}+H_{\alpha}\left(\left(X^{*}-x^{*}\right)^{+}\right) .
$$

The chain of equalities above together with item (a) implies that $\left(x^{*}, X^{*}\right) \in$ $\arg \min _{(x, X) \in \mathbb{R} \times \mathcal{X}}\left\{x+H_{\alpha}\left((X-x)^{+}\right)\right\}$.

Proof of Proposition 4.2. This proof can be driven as the one done by Bertsimas et al. ([6]; Proposition 4) in the case of CVaR. We will enclose the proof in the general $\left(\pi_{\alpha}\right)$ case just for completeness.

Consider two arbitrary points $\left(r_{1}, \pi_{\alpha}\left(X^{*}\left(r_{1}\right)\right)\right)$ and $\left(r_{2}, \pi_{\alpha}\left(X^{*}\left(r_{2}\right)\right)\right)$ on the frontier curve and take $\beta \in(0,1)$.

Set $r_{\beta}=\beta r_{1}+(1-\beta) r_{2}$ and set $\mathbf{w}^{*}\left(r_{\beta}\right)$ the optimal portfolio's weights.

Take note that, for $\mathbf{w}^{\beta}=\beta \mathbf{w}^{*}\left(r_{1}\right)+(1-\beta) \mathbf{w}^{*}\left(r_{2}\right)$, the return $X^{\beta}$ of the portfolio with weights $\mathbf{w}^{\beta}$ is given by $X^{\beta}=\mathbf{X} \cdot \mathbf{w}^{\beta}=\beta X^{*}\left(r_{1}\right)+(1-\beta) X^{*}\left(r_{2}\right)$, hence $E\left[X^{\beta}\right]=\beta E\left[X^{*}\left(r_{1}\right)\right]+(1-\beta) E\left[X^{*}\left(r_{2}\right)\right]$. It follows therefore that

$$
\begin{aligned}
\pi_{\alpha}\left(X^{*}\left(r_{\beta}\right)\right) & =\min _{\mathbf{w} \in W: E[\mathbf{X} \cdot \mathbf{w}]=r_{\beta}} \pi_{\alpha}(\mathbf{X} \cdot \mathbf{w}) \\
& \leq \pi_{\alpha}\left(X^{\beta}\right)=\pi_{\alpha}\left(\beta X^{*}\left(r_{1}\right)+(1-\beta) X^{*}\left(r_{2}\right)\right) \\
& \leq \beta \pi_{\alpha}\left(X^{*}\left(r_{1}\right)\right)+(1-\beta) \pi_{\alpha}\left(X^{*}\left(r_{2}\right)\right),
\end{aligned}
$$

where (5.3) is due to the arguments above and (5.4) to the convexity of $\pi_{\alpha}$. Hence the frontier curve is convex in the plane $\left(r, \pi_{\alpha}\right)$.

\section{References}

[1] C. Acerbi. Spectral measures of risk: A coherent representation of subjective risk aversion. Journal of Banking \& Finance, 26/7: 1505-1518, 2002.

[2] P. Artzner, F. Delbaen, J.M. Eber and D. Heath. Coherent measures of risk. Mathematical Finance, 4: 203-228, 1999.

[3] P. Barrieu and N. El Karoui. Inf-convolution of Risk Measures and Optimal Risk Transfer. Finance and Stochastics 9: 269-298, 2005.

[4] F. Bellini and E. Rosazza Gianin. On Haezendonck risk measures. To appear on Journal of Banking and Finance, 2006.

[5] A. Ben-Tal and M. Teboulle. An old-new concept of convex risk measures: the optimized certainty equivalent. To appear on Mathematical Finance, 2006 . 
[6] D. Bertsimas, G.J. Lauprete and A. Samarov. Shortfall as a risk measure: properties, optimization and applications. Journal of Economic Dynamics E Control, 28: 1353-1381, 2004.

[7] M.J. Goovaerts, R. Kaas, J. Dhaene and Q. Tang. Some new classes of consistent risk measures. Insurance: Mathematics and Economics, 34/3: 505-516, 2004.

[8] J. Haezendonck and M. Goovaerts. A new premium calculation principle based on Orlicz norms. Insurance: Mathematics and Economics, 1: 41-53, 1982.

[9] F.R. Hampel. The Influence Curve and Its Role in Robust Estimation. Journal of the American Statistical Association, 69/346: 383-393, 1974.

[10] C.F. Huang and R. Litzenberger. Foundations for Financial Economics. North-Holland, Amsterdam, 1988.

[11] P.J. Huber. Robust Statistics. Wiley, New York, 1981.

[12] M.A. Krasnosel'skii and J.B. Rutickii. Convex Functions and Orlicz Spaces. Noordhoff, Groningen, 1961.

[13] S. Kusuoka. On law invariant coherent risk measures. In S. Kusuoka and T. Maruyama, eds., Advances in Mathematical Economics, Vol. 3, pages 83-95, Tokyo, 2001, Springer.

[14] M.M. Rao and Z.D. Ren. Theory of Orlicz Spaces. New York: Marcel Dekker Inc, 1991.

[15] R.T. Rockafellar. Convex Analysis. Princeton University Press, 1970.

[16] R.T. Rockafellar and S. Uryasev. Optimization of conditional value-at-risk. Journal of Risk, 2: 21-41, 2000.

[17] R.T. Rockafellar and S. Uryasev. Conditional value-at-risk for general loss distributions. Journal of Banking and Finance, 26/7: 1443-1471, 2002.

[18] R. Serfling. Approximation theorems of mathematical statistics. Wiley, New York, 2001.

Fabio Bellini

Dipartimento di Metodi Quantitativi

Università di Milano Bicocca

Piazza dell'Ateneo Nuovo 1

20126 Milano, Italy

fabio.bellini@unimib.it
Emanuela Rosazza Gianin

Dipartimento di Matematica e Statistica

Università di Napoli "Federico II"

Monte S. Angelo - Via Cinthia

80126 Napoli, Italy

grosazza@unina.it 

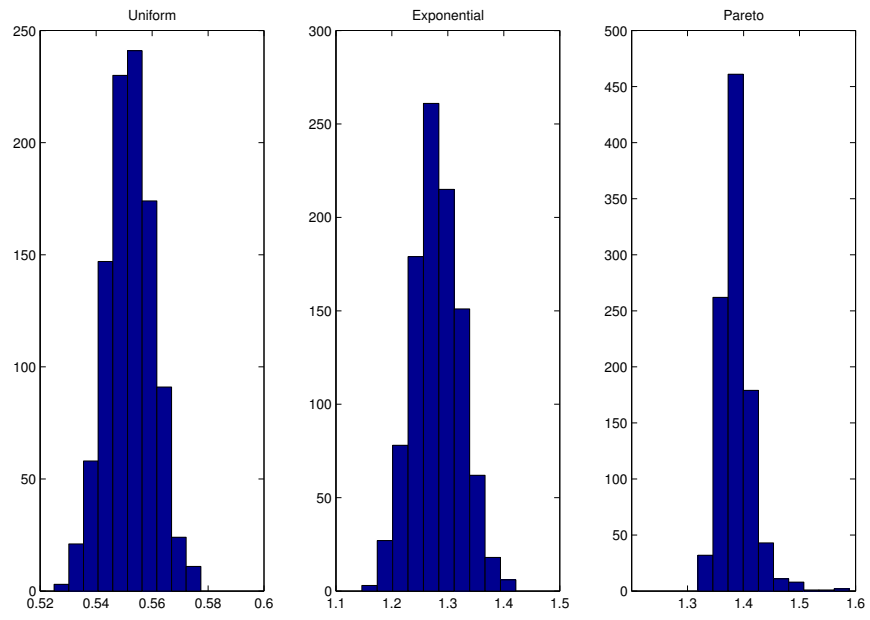

Figure 5.1: The distribution of $\widehat{H}_{0}$ in the Uniform case (left), in the Exponential case (center) and in the Pareto case (right).
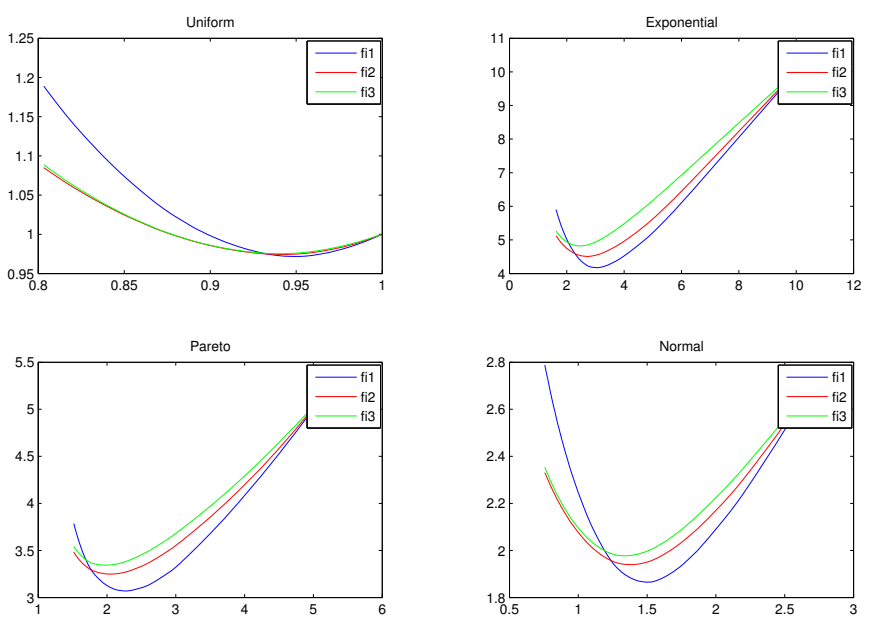

Figure 5.2: Numerical computation of the functions $\widehat{\pi}_{0.95}(X, x)$ on simulated data in the Uniform case (upper left), in the Exponential case (upper right), in the Pareto case (lower left) and in the Normal case (lower right) for three different Young functions. 

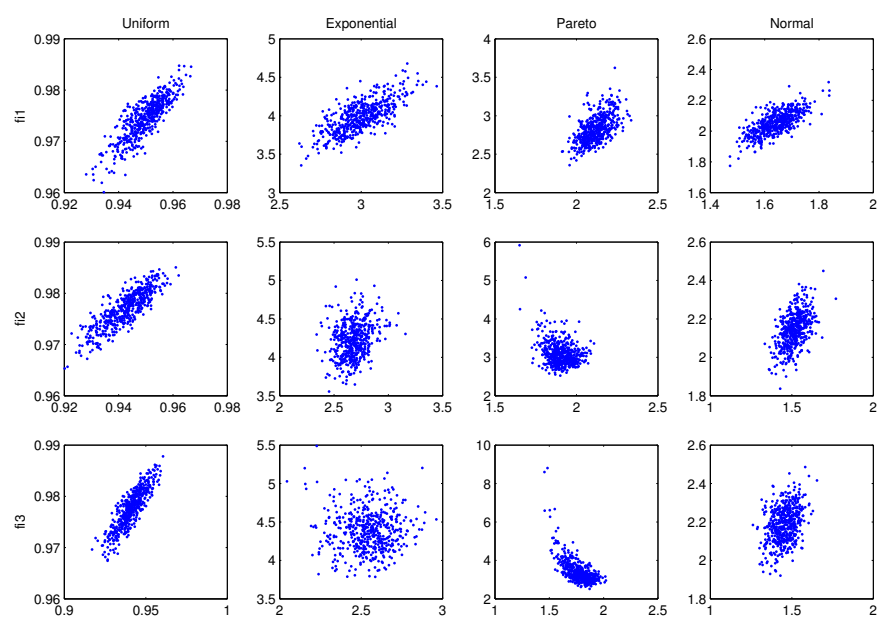

Figure 5.3: The joint distribution of $x^{*}$ and $\hat{\pi}_{0.95}(X)$ on simulated data with respect to different Young functions.
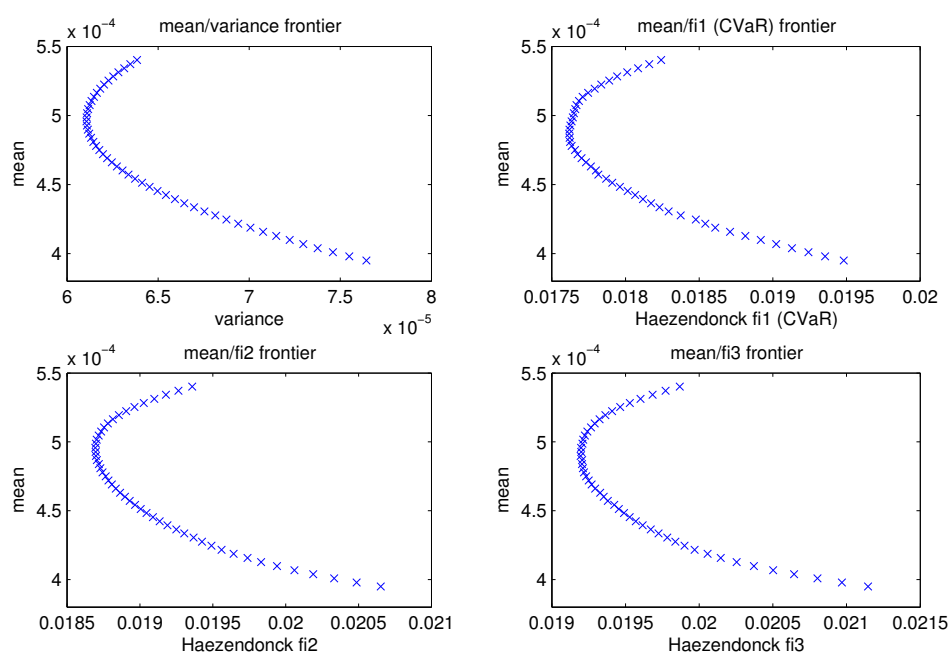

Figure 5.4: Efficient frontiers in the four considered cases: mean/variance (upper left), mean/CVaR (upper right), mean/fi2 (lower left) and mean/fi3 (lower right). 

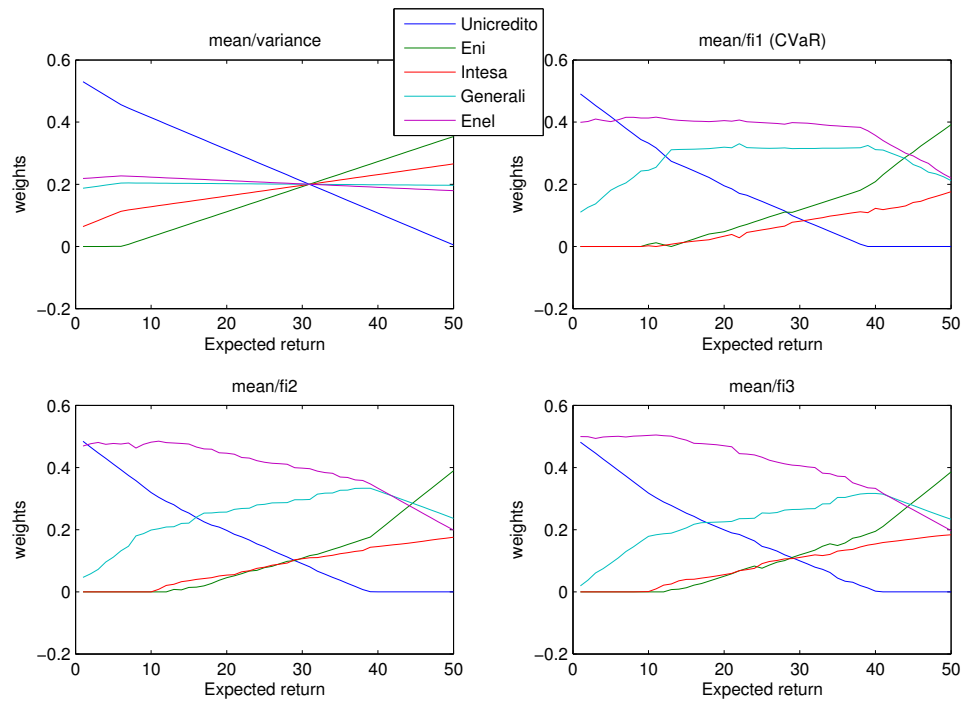

Figure 5.5: Optimal portfolios' weights for different target returns $r_{p}$ in the four considered cases: mean/variance (upper left), mean/CVaR (upper right), mean/fi2 (lower left) and mean/fi3 (lower right). 